\title{
Relations entre certaines propriétés du bois et les fentes des arbres sur pied chez Abies grandis (Lindl.)
}

\author{
B. BOULET-GERCOURT et G. NEPVEU \\ I.N.R.A., Station de recherches sur la qualité des bois \\ Centre de Recherches de Nancy, Champenoux, F 54280 Seichamps
}

\section{Résumé}

L'étude a porté sur un échantillonnage de 48 Abies grandis âgés de 40 ans environ comportant 24 arbres fendus et 24 arbres témoins appariés en circonférence avec les précédents. Le but du travail était de comparer certaines propriétés du bois des deux groupes.

Les différentes caractéristiques du bois prises en considération étaient les suivantes : angle du fil du bois, infradensité du bois, variations de la densité du bois dans le cerne ou entre cernes.

On a obtenu les résultats suivants :

- à diamètre égal, les arbres fendus ont la même hauteur, le même volume, la même forme, la même excentricitê du cceur que les arbres non fendus.

Toujours à diamètre égal :

- les arbres fendus ont (à $7,50 \mathrm{~m}$ de hauteur) un angle du fil du bois supérieur à celui des arbres non fendus (tabl. 1, fig. 3);

- l'infradensité du bois du cerne 1969 (année où la majorité des fentes se sont produites) est plus faible dans la population d'arbres fendus (tabl. 2a);

- l'infradensité des arbres fendus est significativement plus faible que celle des arbres non fendus, ce de façon régulière sur la section transversale de la tige (tabl. 2b) ; les mesures faites à la souche confirment les résultats trouvés à $6,50 \mathrm{~m}$ de hauteur (tabl. 2c);

- l'étude microdensitométrique a permis de mettre en évidence un bois final significativement moins important (en pourcentage et surtout en largeur) chez les arbres fendus (tabl. 3), de confirmer une relative régularité des écarts de densité entre les deux populations sur la section transversale de la tige (fig. 4) et de localiser ces écarts de façon précise à l'intérieur du cerne, pour l'essentiel à la limite du bois de printemps et du bois d'été (fig. 5).

La notation des fentes a permis de constater certaines relations entre le climat et l'apparition du défaut.

La structure du bois des arbres fendus semble se distinguer plus nettement de celle des arbres non fendus lors des années à risque. sujet.

Tous ces résultats sont relativement cohérents avec ceux des rares publications existant sur le

Les auteurs concluent en proposant des moyens concrets pour réduire le risque d'apparition du défaut et en suggérant diverses voies de recherche pour étudier plus à fond le phénomène.

Mots clés: Fente du tronc, masse volumique, microdensitométrie, fibre torse, Abies grandis. 


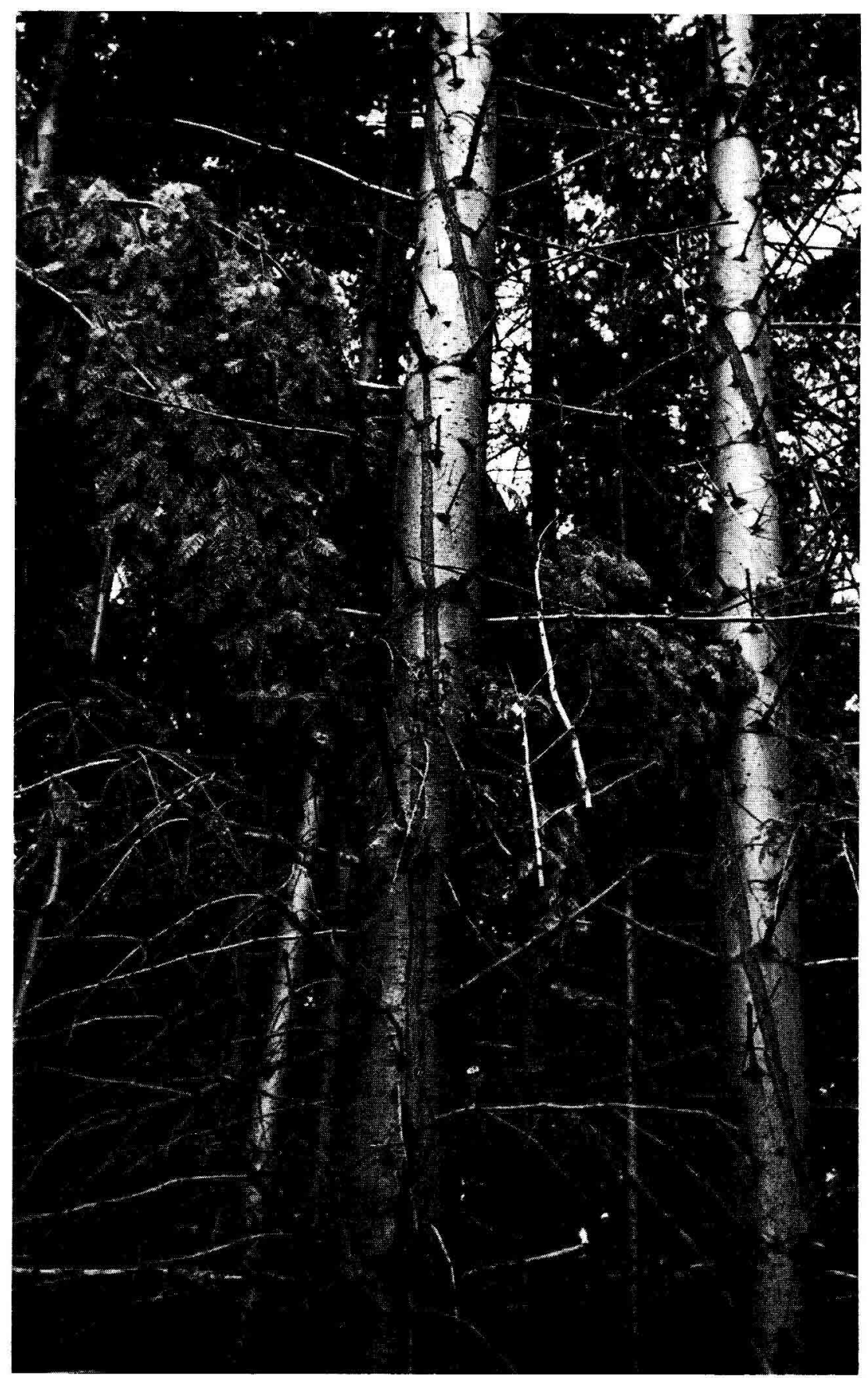

FIG. 1

Traces de fentes recouvertes sur des Abies grandis de la présente étude. Marks of covered cracks in grand firs studied here. 


\section{Introduction}

\subsection{Généralités}

Le défaut des fentes dites de sécheresse, que l'on peut rencontrer chez un certain nombre de résineux, reste à ce jour peu connu car peu étudié : les travaux de DAY (1954) et de Dietrichson et al. (1985), les plus importants, ont été menés respectivement en Grande-Bretagne sur plusieurs espèces de conifères et en Norvège sur Picea abies (L.). Depuis quelques années la question revient sur le devant de la scène car on rencontre de plus en plus fréquemment des arbres fendus sur pied dans les plantations, notamment celles d'Abies grandis (Lindl.) et de Picea abies (L.).

Si l'on excepte le travail de Monchaux et de Nepveu (1986) qui a porté sur deux plantations d'Epicéa commun, aucune étude n'avait encore été entreprise qui soit centrée sur les principaux paramètres de structure du bois (notamment la densité du bois au sens large) en liaison avec ce problème des fentes: l'article présenté ici comble donc une lacune.

On doit ajouter qu'il est en grande partie repris d'un document beaucoup plus complet et volumineux (BOULET-GERCOURT, 1986) auquel on se reportera notamment pour les détails d'ordre méthodologique.

\subsection{Description des fentes}

\subsection{Aspect extérieur}

Sauf si la fente s'est ouverte dans l'année, ce que l'on observe (fig. 1) est en fait la cicatrice d'une fente survenue antérieurement; cette cicatrice figure l'angle du fil du bois au moment où l'événement est intervenu. Ceci explique que, si l'on ôte l'écorce sous une vieille cicatrice, l'angle du fil du bois observé au niveau du cambium soit différent de celui indiqué par la cicatrice ; le premier est d'ailleurs beaucoup plus fermé que le second car de façon générale l'angle du fil a tendance à diminuer avec l'âge. Notons également qu'en ce qui nous concerne nous n'avons observé que des angles positifs (dans le sens trigonométrique).

On constate par ailleurs une extrême diversité suivant les peuplements et les individus quant à la longueur des fentes, leur nombre, leur position sur le tronc. Dans notre cas, le pourcentage de longueur du tronc fendue par rapport à la hauteur de l'arbre variait de 10 à 44 p. 100 ; les fentes commençaient au minimum à $2,2 \mathrm{~m}$ de hauteur pour se terminer à $20,1 \mathrm{~m}$. Il y avait 1 à 8 fentes par arbre.

\subsection{Description interne}

La figure 2 présente le positif d'une radiographie obtenue à partir d'une plaquette de bois de $5 \mathrm{~mm}$ d'épaisseur suivant une section transversale d'une tige d'Abies grandis.

On observera :

- la direction radiale de la fente ;

- la réaction du cambium qui produit un cal de cicatrisation l'année suivant l'ouverture de la fente. Cette réaction rapide explique qu'en ôtant l'écorce au niveau d'une cicatrice on trouve un bois sain au niveau du cambium; 


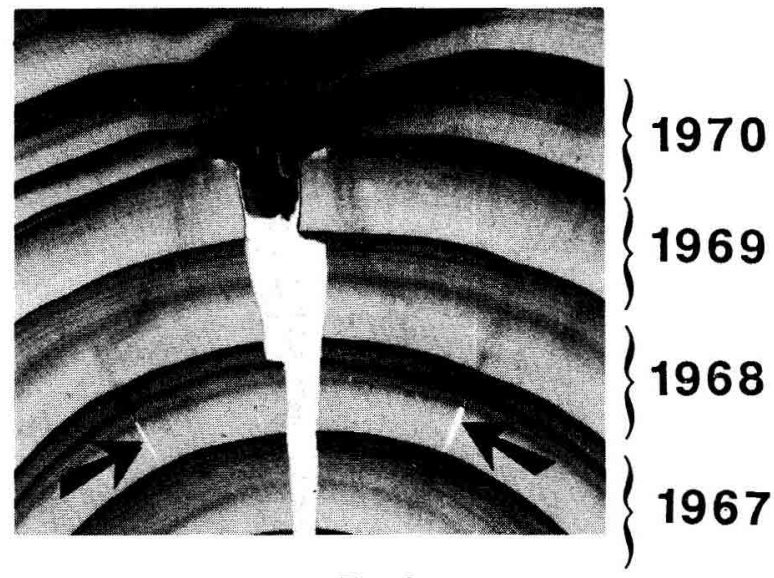

FIG. 2

Aspect interne d'une fente (coupe transversale).

Positif de radiographie d'une plaquetie de 5 mon d'épaisseur.

NB : la fente est intervenue en 1969; deux microfentes sont visibles dans le cerne 1967.

Internal aspect of a crack (transversal cutting).

Radiographic positive of a $5 \mathrm{~mm}$ thick cutting.

NB : the crack occured in $1969 ; 2$ microcracks can be seen in ring 1967.

- un léger amincissement du cerne près de la fente ce qui indique que cet événement s'est produit à la fin de la saison de végétation avant que l'activité cambiale soit arrêtée.

Si lannée de la formation de la fente peut être connue sans aucune ambiguïté (il faut toujours abattre l'arbre) et la période dans l'année située très schématiquement vers la fin de la saison de végétation, il est d'autre part extrêmement probable qu'il y ait une relation directe entre l'apparition de ces fentes et l'existence d'une période à fort déficit d'approvisionnement en eau des arbres comme l'indiquent les auteurs des 4 documents cités plus haut. D'où le terme de fentes de sécheresse.

Il convient enfin d'ajouter qu'à côté de ces fentes de « sécheresse » que l'on vient de décrire, un nombre assez important de très petites fentes (microfentes), le plus souvent limitées au cerne, a été observé dans l'échantillonnage retenu ici. Deux microfentes sont ainsi bien visibles sur la figure 2 dans le cerne 1967. Bien que limitées dans le sens radial, ces microfentes peuvent être très développées dans le sens longitudinal ce qui pourrait poser des problèmes pour l'usinage du bois. Elles ont aussi été signalées chez l'Epicéa commun (Monchaux et Nepveu, 1986, op. cit.) et chez le Douglas (Polge, 1982 et 1984 ; Melin, 1984). Sur Abies grandis, Boulet-Gercourt (1986, op. cit.) en a dénombré jusqu'à 89 sur un disque comptant 23 cernes !

La présence de ces microfentes a d'ailleurs été mise en relation avec la distribution fine de densité du bois dans le cerne. 


\subsection{Gravité du défaut}

Il s'agit d'un défaut grave pour plusieurs raisons :

- au niveau des peuplements, le pourcentage d'arbres fendus peut être élevé. Sur Abies grandis en Normandie, LANGLET (1985) a trouvé des peuplements touchés à plus de 50 p. 100 mais surtout, la « probabilité de fente » croît régulièrement à âge égal, avec la grosseur des arbres. Schématiquement, ce sont donc les arbres les plus gros des plantations les plus productives qui sont touchés ;

- au niveau individuel, la dépréciation est très forte à cause de l'enroulement de la fente dans le bois, des altérations du bois (pourritures), de la réduction des propriétés mécaniques qui en résulte... On doit ajouter que la longueur de tige effectivement fendue est souvent importante.

\subsection{Buts de cet article}

L'étude dont cet article rend compte a été effectuée à partir d'un échantillon d'arbres choisis dans une parcelle où plus de $20 \mathrm{p} .100$ des individus présentaient une ou plusieurs fentes.

Notre démarche a consisté à considérer ces deux groupes (arbres non fendus et arbres fendus) comme deux populations a priori distinctes et à chercher une réponse aux questions suivantes:

- peut-on discriminer ces deux populations à l'aide de certains paramètres décrivant la structure du bois?

- dans l'affirmative, peut-on en tirer quelques principes d'action pour tenter à terme de diminuer le risque d'apparition de ce défaut dans les peuplements? Ce point sera évoqué dans la conclusion.

Le choix des paramètres étudiés s'est porté sur l'angle du fil, l'infradensité et les composantes de la densité du bois obtenues en analyse microdensitométrique et décrivant la distribution "fine » de densité du bois dans le cerne. Le paramètre « densité du bois » était a priori un critère de choix du fait de ses bonnes liaisons avec les propriétés mécaniques du bois et de la simplicité de sa mesure y compris de façon non destructive.

Dans un souci de cohérence avec notre démarche, nous avons choisi :

- d'accorder une attention particulière à des caractéristiques «synthétiques " par arbre : l'utilisation privilégiée de variables «moyennes» intégrant tous les cernes a répondu à cette préoccupation (angle du fil moyen, infradensité globale, cerne moyen pour l'étude microdensitométrique) ;

- de travailler à circonférence égale entre les deux sous-échantillons (individus non fendus et fendus) : ceci a dicté le choix de couples appariés sur la circonférence à $1,30 \mathrm{~m}$.

En ce qui concerne ce second point, un autre choix eût été possible : il aurait consisté à tirer au hasard les arbres témoins dans l'ensemble du peuplement et les arbres fendus dans la sous-population des arbres fendus. Inévitablement, les arbres fendus eussent été plus gros car l'effet « taille de l'arbre " est celui qui "sort " en premier, et ce de façon incontestable, lorsque l'on cherche à expliquer la sensibilité au 
défaut considéré ici, à l'intérieur d'un peuplement équienne. Une telle démarche qui présente par ailleurs des avantages incontestables aurait toutefois nécessité de disposer d'un nombre d'arbres beaucoup plus élevé notamment pour faire la part de ce qui revenait aux différences de vitesse de croissance dans les écarts de propriétés du bois notés entre les 2 populations.

\section{Matériel et méthodes}

\subsection{Echantillonnage et prélèvement du matériel de base}

\subsection{Echantillonnage}

La parcelle, dont seule une partie (ni éclaircie, ni élaguée) a été utilisée, se trouve en forêt d'Amance (Meurthe-et-Moselle). La plantation a été effectuée en 1945-1946 à $2 \mathrm{~m} \times 2,50 \mathrm{~m}$.

Pour des raisons pratiques, il n'était pas possible de prélever des éprouvettes à moins de $6 \mathrm{~m}$ de la souche, ce qui explique la suite.

Le principe du tirage de l'échantillon a été le suivant :

- choix au hasard d'un arbre fendu avec au moins une fente présente entre $6,50 \mathrm{~m}$ et $7,50 \mathrm{~m}$ de hauteur ;

- choix pour chaque tige fendue d'un " jumeau " totalement exempt de fente, de même circonférence à $1,30 \mathrm{~m}$ et le plus proche possible du précédent sur le terrain.

En définitive 48 arbres (soit 24 couples) ont été choisis. Leur hauteur totale moyenne était de $26,5 \mathrm{~m}$ pour une circonférence moyenne à $1 \mathrm{~m} \mathrm{de} 106 \mathrm{~cm}$. Les hauteurs individuelles variaient de 23 à $29 \mathrm{~m}$ et les circonférences de 72 à $141 \mathrm{~cm}$.

Nous précisons qu'à ce niveau, le premier cerne visible à la moelle correspondait en général à l’année 1965 ou 1964.

\subsection{Mesures avant prélèvement}

Juste après l'abattage intervenu en décembre 1985, nous avons mesuré sur chaque arbre sa hauteur totale et la circonférence de la tige tous les mètres à partir de la souche (jusqu’à la découpe $20 \mathrm{~cm}$ de circonférence).

\subsection{Prélèvement du matériel de base}

Compte tenu de la contrainte évoquée au 2.11 ., on a choisi de prélever sur la coupe dès après l'abattage :

- un disque d'environ $2 \mathrm{~cm}$ d'épaisseur à $6,30 \mathrm{~m}$ de hauteur mis aussitôt sous plastique pour mesure du taux d'humidité : disque $\mathrm{H}$;

- un disque de $4 \mathrm{~cm}$ d'épaisseur à la souche pour mesures d'infradensité : disque IS ;

- un billon de $1,40 \mathrm{~m}$ de long entre les niveaux $6,30 \mathrm{~m}$ et $7,70 \mathrm{~m}$, dans lequel on a ensuite découpé :

- trois disques denviron $1 \mathrm{~cm}$ utilisés par la suite pour observer et dater les fentes puis mesurer les infradensités: disques I1, 12, I3, 
- un disque d'environ $8 \mathrm{~cm}$ d'épaisseur dont on a tiré ultérieurement une barrette pour microdensitométrie : disque $\mathrm{DE}$,

- un billon de $40 \mathrm{~cm}$ de long qui a servi pour les mesures d'angle de fil et le calcul d'un indice d'excentricité de la moelle : billon $F$.

\subsection{Mesures réalisées}

\subsection{Caractéristiques dendrométriques des tiges}

- volumes « tige » à partir du cubage ;

- trois coefficients de décroissance métrique entre $1 \mathrm{~m}$ et le sommet, entre $1 \mathrm{~m}$ et $8 \mathrm{~m}$, entre $8 \mathrm{~m}$ et le sommet ;

- coefficient de forme suivant la formule classique, la circonférence à $1 \mathrm{~m}$ remplaçant celle à $1,30 \mathrm{~m}$.

\subsection{Excentricité du cour}

L'une des faces du billon (cf. 2.13.) a servi à reporter sur calque le contour de cette face ainsi que celui du cerne 1969 (année où la majorité des fentes se sont produites ; voir 3.11.) ; on a mesuré sur chacun de ces 2 contours, le plus grand rayon et son opposé. Le rapport des 2 a défini un indice d'excentricité du cœur qu'on a établi pour 1969 et 1985.

\subsection{Angle du fil du bois}

Le principe de base de la mesure en est simple. Un disque est fendu à l'aide d'une presse à partir d'une amorce de fente rectiligne réalisée sur l'une des faces et passant par la moelle. Il en résulte sur l'autre face un bord sinueux reflétant les variations de l'angle du fil depuis la moelle jusqu'à l'écorce. utilisé.

Un disque exempt de nouds de $7 \mathrm{~cm}$ d'épaisseur, tiré du billon $\mathrm{F}$ (cf. 2.13) a été

On dispose pour chaque cerne d'une valeur d'angle de fil moyen pour l'arbre (moyenne arithmétique sur tous les cernes).

\subsection{Infradensité du bois (poids anhydre/volume saturé)}

Trois études différentes ont été conduites :

- dans la première, seul le cerne 1969, année à "fentes", a été considéré. Pour chaque arbre, l'infradensité considérée est la moyenne de 12 observations relatives à des échantillons prélevés dans le cerne 1969 sur le disque I1 en évitant, le cas échéant, la zone au voisinage de la fente qui risquait d'être altérée ;

- dans la seconde, on souhaitait comparer l'infradensité moyenne des 2 populations en distinguant quatre zones depuis la moelle jusqu'à l'écorce. Sur le disque I2, on découpait pour ce faire 2 secteurs et sur chaque secteur 3 zones corrèspondant à 5 cernes en commençant à partir de la moelle et une $4^{c}$ zone proche de l'écorce comportant un nombre variable de cernes. Pour chaque arbre et chaque zone, l'infradensité retenue est donc la moyenne de 2 observations ;

- dans la troisième étude, menée à la souche, on cherchait essentiellement à voir si les résultats trouvés à $7 \mathrm{~m}$ de hauteur étaient confirmés au niveau du sol, ce qui 
serait tout à fait intéressant pour des mesures non destructives. Pour ce faire on découpait, sur le disque IS, deux secteurs et sur chaque secteur, deux zones; la première zone correspondait aux 15 premiers cernes depuis la moelle, la seconde aux cernes restant jusqu'à l'écorce. Pour chaque arbre et chaque zone, l'infradensité retenue était donc une moyenne de 2 observations.

Pour ces trois études, on a utilisé la méthode de saturation intégrale (KEYLwERTH, 1954) pour mesurer l'infradensité ; le poids saturé était obtenu par ébullition dans l'eau durant 24 heures.

\subsection{Distribution "fine» de la densité du bois de cerne}

Du disque DE (cf. 2.13) a été tiré un bloc opposé à la fente sur les rondelles fendues. On y a découpé une barrette radiale pour radiographie. L'exploration au microdensitomètre de ces radiographies a permis d'obtenir pour chaque cerne 29 variables (largeur de cerne, densité minimum de cerne, ...). Un cerne moyen a ensuite été défini pour chaque arbre en prenant pour chacune de ces 29 variables la moyenne arithmétique des valeurs prises pour chaque cerne (cernes 1967 à 1985).

\subsection{Traitement statistique réalisé}

Pour chacun des caractères mesurés sur les 48 arbres, nous avons effectué des analyses de variance à 2 facteurs croisés sans interaction. Les effets contrôlés étaient les effets «population» (arbres fendus et arbres non fendus) à 1 degré de liberté et l'effet « couple " à 23 degrés de liberté. La variance résiduelle avait 24 degrés de liberté. Les $F$ pris en compte pour tester la signification des éventuelles différences entre les arbres "fendus" et «non fendus » avaient done 1 et 24 degrés de liberté.

Dans la présentation des résultats (tableaux et figures), les symboles suivants seront utilisés :

NS : test $F$ non significatif

* : test $\mathrm{F}$ significatif au seuil de 5 p. 100

${ }^{* * *}$ : test F significatif au seuil de 1 p. 100

\section{Résultats et discussion}

\subsection{Premiers résultats}

\subsection{Dates de formation des fentes}

Sur les 24 arbres fendus, 73 fentes ont été localisées dont 43 ont pu être datées ; parmi ces dernières, on a trouvé une ou plusieurs fentes datées de :

1969 sur 23 arbres

1967 sur 6 arbres

1976 sur 1 arbre

1978 sur 1 arbre 
Ceci met particulièrement en valeur l'année 1969. C'est pourquoi nous avons cherché à savoir en quoi son climat pouvait différer des autres années. Nous avons pu constater que si 1969 n'était pas une année particulièrement sèche, elle se distinguait des autres par un déficit hydrique assez prononcé sur trois mois couvrant la fin de l'été et le début de l'automne (août, septembre, octobre). Ceci est à rapprocher du fait que, comme indiqué en introduction, la fente se forme vers la fin de la saison de végétation avant que l'activité cambiale ait cessé.

Bien que la responsabilité d'une sécheresse estivale tardive soit aussi incriminée dans plusieurs articles cités en bibliographie, nous resterons prudents sur notre interprétation en rappelant au lecteur qu'elle ne concerne qu'un peuplement équienne et qu'au niveau des principales mesures $(6,50 \mathrm{~m})$ l'année 1969 correspondait à un bois juvénile à densité particulièrement faible.

\subsection{Taux d'humidité du bois}

Aucune différence significative entre les 2 populations (arbres non fendus et arbres fendus) n'apparaît.

Ce résultat doit être considéré avec la plus grande prudence car la mesure faite en décembre 1985 n'a sans doute pas été réalisée à un moment où l'humidité du bois était minimum et où en principe les fentes doivent se produire.

\subsection{Caractéristiques dendrométriques}

Entre ces deux populations, identiques quant à leur circonférence à $1,30 \mathrm{~m}$, on n'a trouvé aucune différence significative pour la hauteur, le volume, les coefficients de décroissance métrique et le coefficient de forme.

\subsection{Excentricité de la moelle}

Aucune différence significative n'apparaît entre les deux populations.

\subsection{Angle du fil du bois}

Avec la convention de choisir comme angle positif, l'angle de fil habituel sur les arbres (fig. 1), les résultats sont indiqués dans le tableau 1.

\section{TABLEAU 1}

Angle moyen du fil du bois pour les arbres fendus et les arbres témoins.

Mean of spiral grain in cracked trees and control trees.

Valeur du $F$ et niveau de signification $=F$ value and significance level

Arbres témoins $=$ control trees

Arbres fendus $=$ cracked trees

Angle du fil du bois = spiral grain

\begin{tabular}{l|c|c|c}
\hline & Arbres témoins & Arbres fendus & $\begin{array}{c}\text { Valeur du F } \\
\text { et niveau } \\
\text { de signification }\end{array}$ \\
\cline { 2 - 4 } Angle du fil du bois (degrés) & 2,3 & 3,0 & $3,6 \mathrm{NS}$ \\
\hline \hline
\end{tabular}



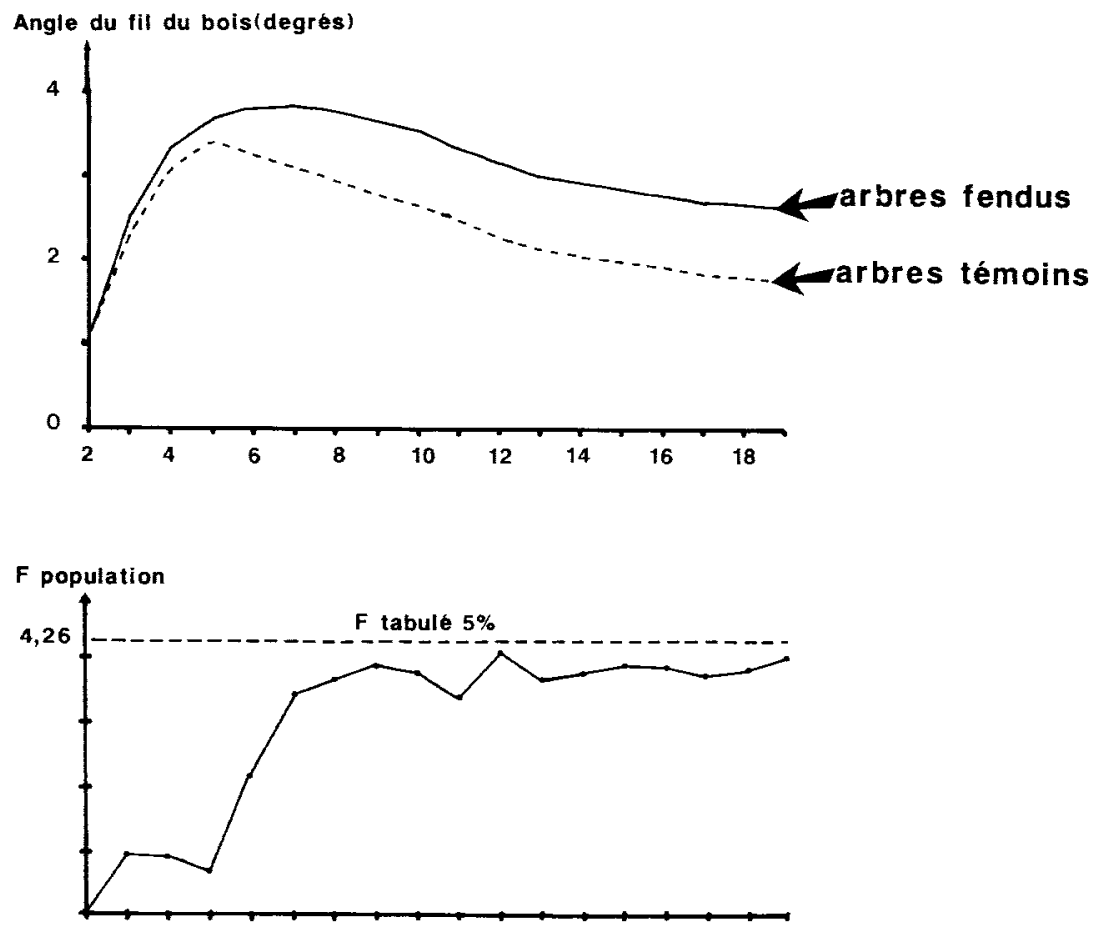

NUMERO DE CERNE DEPUS LA MOELLE

Fig. 3

Evolution de l'angle du fil du bois en fonction de l'âge du cerne compté à partir de la moelle pour les arbres fendus et les arbres témoins.

Variation of spiral grain in relation with ring age from the pith for cracked trees and control trees.

Arbres fendus $=$ cracked trees

Arbres témoins $=$ control trees

Age du cerne depuis la moelle = ring number from the pith

Angle du fil du bois = spiral grain

La différence entre les deux populations est donc de $30 \mathrm{p} .100$ et la valeur du $\mathrm{F}$ proche du seuil de 5 p. $100(4,26)$ bien que non significative.

Si l'on examine l'évolution de l'angle du fil suivant le numéro de cerne à partir de la moelle (fig. 3), on constate un net décalage entre les deux courbes à partir du $7^{\circ}$ cerne depuis la moelle. Il semble donc tout à fait réaliste de considérer que les valeurs de l'angle du fil du bois, mesuré à environ 7,50 $\mathrm{m}$ de hauteur sont supérieures chez les arbres fendus bien que, nous le répétons, les $F$ observés âge par âge et reportés sur la figure 3 n'atteignent pas la valeur-seuil à 5 p. 100 .

En nous gardant de toute extrapolation audacieuse, nous ne pouvons pas ne pas rapprocher ces résultats de ceux de Ferrand (1982) qui avait montré que les Hêtres à fibre torse avaient un niveau de contraintes internes beaucoup plus élevé que les autres ; ceci constituant d'ailleurs une démonstration expérimentale des calculs théori- 
ques de ArCher (1979). Nous ne sommes donc pas autrement surpris de nos résultats puisqu'il n'est pas a priori étonnant que les arbres ayant davantage de contraintes internes (du fait de leur fil tors) soient plus sensibles que les autres à la fente.

Ces résultats qui confirment une fois de plus le rôle important de l'angle du fil du bois pour la qualité du bois rend plus que jamais nécessaire la mise au point d'une méthodologie fiable et rapide de mesure de ce caractère.

\subsection{Infradensité du bois}

Les résultats sont regroupés dans le tableau 2.

TABLEAU 2

Comparaison de l'infradensité du bois des arbres fendus et des arbres témoins.

Résultats des trois études d'infradensité.

Comparison for basic wood density in cracked trees and control trees.

Results for the 3 studies related with basic density.

\section{TABLEAU $2 \mathrm{a}$}

Première étude : Infradensité du cerne 1969 (à $6,50 \mathrm{~m}$ de hauteur) en $\mathrm{g} / \mathrm{dm} \mathrm{m}^{3}$.

First study: Basic density in ring 1969 (at $6,50 \mathrm{~m}$ level) in $\mathrm{g} / \mathrm{dm}^{3}$.

\begin{tabular}{c|c|c|c}
\hline Infradensité & Arbres témoins & Arbres fendus & $\begin{array}{c}\text { Valeur du F et } \\
\text { niveau de signification }\end{array}$ \\
\hline Cerne 1969 & 288 & 269 & $19,9 * *$ \\
\hline
\end{tabular}

TABLEAU $2 b$

Seconde étude : Infradensité par zone (à $6.50 \mathrm{~m}$ de hauteur) en $\mathrm{g} / \mathrm{dm}^{3}$.

Second study: Basic density in each zone $\left(a t 6,50 \mathrm{~m}\right.$ level) in $\mathrm{g} / \mathrm{dm}^{3}$.

\begin{tabular}{c|c|c|c}
\hline Infradensité & Arbres témoins & Arbres fendus & $\begin{array}{c}\text { Valeur du F et } \\
\text { niveau de signification }\end{array}$ \\
\hline $\begin{array}{c}\text { Zone 1 } \\
\text { (cernes 1 à 5) }\end{array}$ & 285 & 267 & $20,2^{* *}$ \\
\hline $\begin{array}{c}\text { Zone 2 } \\
\text { (cernes 6 à 10) }\end{array}$ & 292 & 272 & $21,1^{* *}$ \\
\hline $\begin{array}{c}\text { Zone 3 } \\
\text { (cernes 11 à 15) }\end{array}$ & 296 & 278 & $10,7^{* *}$ \\
\hline $\begin{array}{c}\text { Zone juvénile } \\
\text { (cernes 1 à 15) }\end{array}$ & 293 & 273 & $21,4^{* *}$ \\
\hline $\begin{array}{c}\text { Zone 4 } \\
\text { (cerne 16 à l'écorce) }\end{array}$ & 321 & 303 & $10,9^{* *}$ \\
\hline $\begin{array}{c}\text { Ensemble } \\
\text { (cerne 1 à l'écorce) }\end{array}$ & 301 & 282 & $18,4^{* *}$ \\
\hline \hline
\end{tabular}


TABLEAU $2 \mathrm{c}$

Troisième étude: Infradensité à la souche en $\mathrm{g} / \mathrm{dm}^{3}$.

Third study: Basic density at the bottom in $\mathrm{g} / \mathrm{dm}^{3}$.

Valeur du $F$ et niveau de signification $=F$ value and significance level

Arbres témoins $=$ control trees

Arbres fendus $=$ cracked trees

Cerne $=$ ring

Ecorce $=$ bark

\begin{tabular}{c|c|c|c}
\hline Infradensité & Arbres témoins & Arbres fendus & $\begin{array}{c}\text { Valeur du F et } \\
\text { niveau de signification }\end{array}$ \\
\hline $\begin{array}{c}\text { Zone juvénile } \\
\text { (cernes 1 à 15) }\end{array}$ & 370 & 347 & $12,1^{* *}$ \\
\hline $\begin{array}{c}\text { Zone adulte } \\
\text { (cerne 16 à l'écorce) }\end{array}$ & 334 & 317 & $7,5^{* *}$ \\
\hline $\begin{array}{c}\text { Ensemble } \\
\text { (cerne 1 à l'écorce) }\end{array}$ & 340 & 324 & $7,9^{* *}$ \\
\hline
\end{tabular}

- L'infradensité moyenne des arbres fendus est inférieure dans toutes les zones et ce, très significativement. Globalement, c'est-à-dire sur le rayon, l'écart est de 6,7 p. 100 bien que la largeur de cerne ait été supérieure de 6,4 p. 100 chez les nonfendus. On notera que les 2 populations se séparent particulièrement bien au voisinage de la moelle (zones 1 et 2) là où la largeur de cerne est importante et la densité du bois faible (voir fig. 4). L'écart de densité entre les deux groupes se retrouve au niveau de cerne 1969. Tous ces résultats sont très voisins de ceux de Monchaux et Nepveu (1986, op. cit.) qui pour 65 couples d'Epicéa commun trouvaient $290 \mathrm{~g} / \mathrm{dm}^{3}$ pour l'infradensité des arbres fendus et $307 \mathrm{~g} / \mathrm{dm}^{3}$ pour celle des témoins qui leur étaient appariés en circonférence. Avec un peu d'audace, on peut se demander si le seuil d'infradensité de $300 \mathrm{~g} / \mathrm{dm}^{3}$ (environ) n'est pas la limite dangereuse en-deçà de laquelle le phénomène «fente» risque de se produire.

- Si ces différences entre arbres fendus et témoins sont très stables sur le rayon de la tige à $6,50 \mathrm{~m}$ de hauteur, elles le sont également le long du tronc si l'on examine les valeurs à la souche. Ceci indique que si les prélèvements avaient eu lieu à hauteur d'homme, ce qui est plus classique, la discrimination des 2 populations aurait été sans doute assez bonne.

\subsection{Distribution "fine" de densité du bois dans le cerne}

Sur le cerne, les deux populations diffèrent significativement pour la largeur moyenne de cerne, la densité moyenne, le pourcentage et la largeur de bois final.

Aucune différence significative n'apparaît pour les densités minimum de cerne, l'écart-type de densité intracerne, la densité des bois initial et final. 


\title{
TABleaU 3
}

Comparaison de quelques paramètres décrivant la distribution fine de densité du bois dans le cerne pour les arbres fendus et les arbres témoins.

Comparison for some parameters describing wood density distribution in ring for cracked trees and control trees.

\author{
Largeur du cerne $=$ ring width \\ Densité moyenne de cerne = mean density of ring \\ Pourcentage de bois final = late wood ratio \\ Largeur du bois final = late wood width
}

Valeur du $F$ et niveau de signification $=F$ value and significance level

Arbres témoins $=$ control trees

Arbres fendus $=$ cracked trees

\begin{tabular}{l|c|c|c}
\hline & Arbres témoins & Arbres fendus & $\begin{array}{c}\text { Valeur du F } \\
\text { et niveau } \\
\text { dignification }\end{array}$ \\
\cline { 2 - 4 } & 5,82 & 5,45 & $5,2^{*}$ \\
\hline $\begin{array}{l}\text { Largeur de cerne }(\mathrm{mm}) \\
\left(\mathrm{g} / \mathrm{dm}^{3}\right)\end{array}$ & 366 & 346 & $5,8^{*}$ \\
\hline Pourcentage du bois final $(\mathrm{p} .100)$ & 21,7 & 19,4 & $5,2^{*}$ \\
\hline Largeur du bois final $(\mathrm{mm})$ & 1,23 & 1,04 & $14,2^{* *}$ \\
\hline \hline
\end{tabular}

La densité moyenne des arbres fendus est inférieure de 5,8 p. 100 à celle des arbres témoins malgré une largeur moyenne de cerne inférieure de 6,8 p. 100: c'est une situation très semblable à ce que l'on avait trouvé sur l'infradensité globale avec toutefois un niveau de signification plus faible dans ce cas-ci.

Ce qui est nouveau a trait aux deux caractéristiques liées au bois final et particulièrement sa largeur qui est très discriminante. DAY $(1954$, op. cit.) et Dietrichson et al. (1985, op. cit.) avaient tous deux trouvé, essentiellement sur Picea abies et grâce à un travail sur coupes anatomiques, un bois d'été plus faible chez les arbres fendus. La limite du bois d'été avait été déterminée suivant la définition de Mork.

Il n'est pas interdit de penser qu'un bois final plus important constitue une barrière efficace à la propagation de microfissures présentes à l'état andémique dans le bois initial d'Abies grandis les empêchant de ce fait de se transformer en fentes. L'hypothèse n'est pas stupide si l'on songe qu'un bois comme le Douglas qui présente beaucoup de microfentes au voisinage de la moelle est exempt de fentes : ce phénomène pourrait-il s'expliquer par sa texture particulièrement forte empêchant les microfissures de traverser les limites du cerne annuel?

La figure 4 présente l'évolution, en fonction de l'année calendaire, de la largeur de cerne et de la densité moyenne dans les deux groupes, de 1967 à 1985. On notera :

- la bonne «stabilité » des écarts de densité moyenne entre arbres non fendus et arbres fendus et donc l'intérêt de ce paramètre synthétique pour discriminer les deux populations ; 

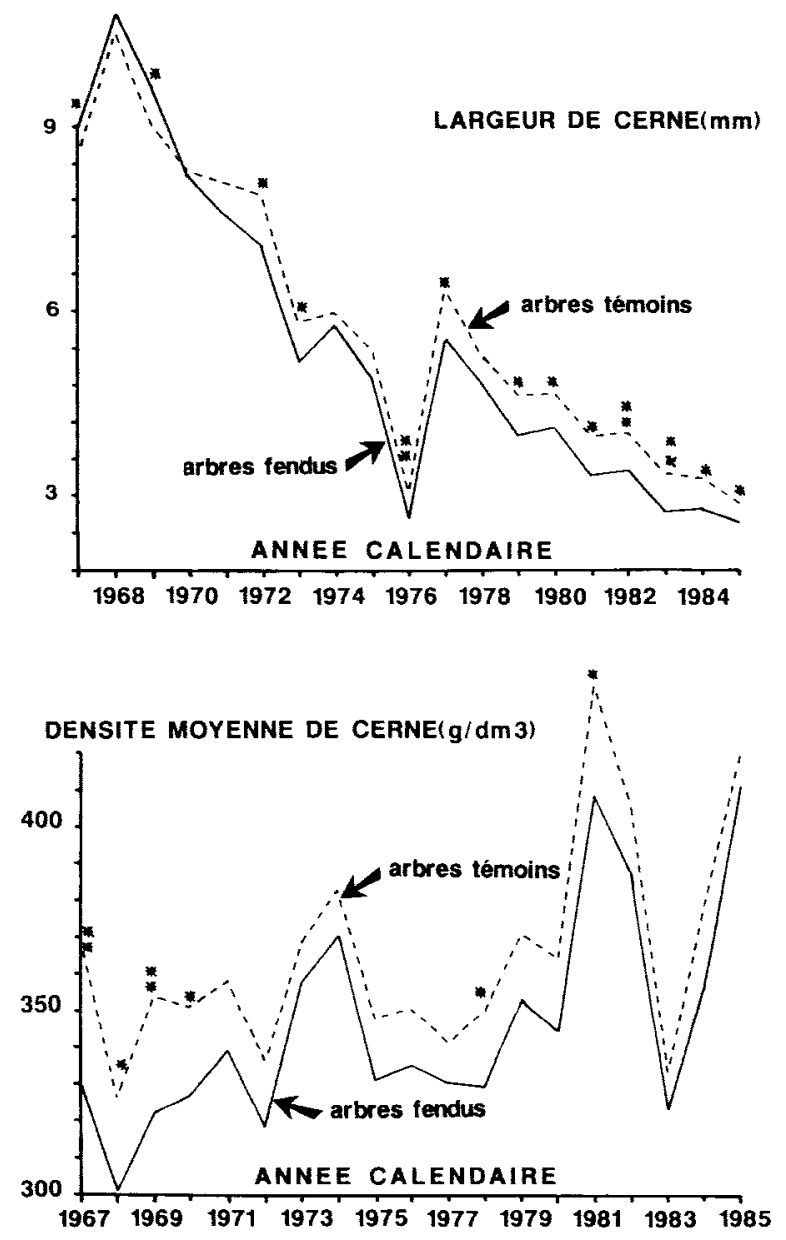

FIG. 4

Evolution suivant l'année calendaire de la largeur de cerne et de la densité du bois pour les arbres fendus et les arbres témoins.

Variation in connexion with calendar year for ring width and wood density in cracked trees and control trees.

Arbres fendus $=$ cracked trees

Arbres témoins $=$ control trees

Largeur de cerne $=$ ring width

Densité moyenne de cerne $=$ mean ring density

Année calendaire $=$ calendar year

- le fait qu'une densité moyenne inférieure chez les arbres fendus soit cependant associée à un cerne moins large sauf dans les premiers cernes. Si la largeur de cerne avait été rigoureusement égale, on peut penser que l'écart de densité aurait été plus grand encore ; 
- que pour les arbres fendus, la densité moyenne dans le cerne 1969 n'est pas la plus basse puisque supérieure à celle des cernes 1968 et 1972 et assez voisine de celle d'autres cernes ;

- que l'écart de densité entre les deux populations est particulièrement significatif lors des «années à fentes». Ainsi sur les 4 années où des fentes ont été relevées $(1967,1969,1976$ et 1978 ; voir 3.11), 3 d'entre elles $(1967,1969$ et 1978) sont parmi les 6 années $(1967,1968,1969,1970,1978$ et 1981) pour lesquelles l'écart de densité du bois est significatif à très significatif. Ce seraient donc les conditions climatiques singulières de ces années à risque qui permettraient aux différences physiologiques entre les deux populations de s'exprimer, déjà par le phénomène de fente, mais aussi par la formation de bois de structures particulièrement différentes ces années-là.

Enfin, les courbes de distribution des densités du bois dans le cerne, représentées pour chacune des 2 populations à la figure 5 , indiquent que les différences de densité ne sont pas réparties de façon uniforme dans le cerne mais localisées sur un «pic » qui recouvre des niveaux de densité intermédiaires, notamment la limite entre fin du bois initial et début du bois final.

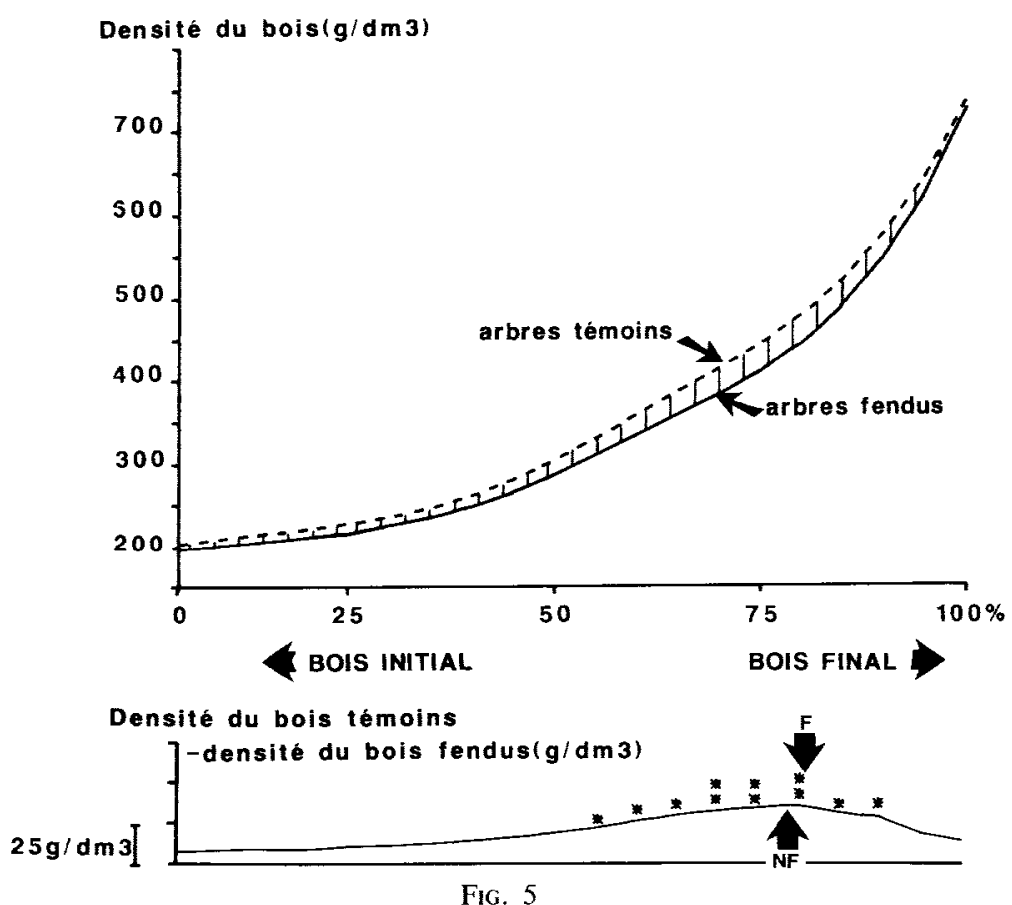

Comparaison des distributions de densité du bois dans le cerne moyen des arbres fendus et des arbres témoins.

Différences de densité du bois entre les deux populations suivant la position dans le cerne.

Comparison for wood density repartition in mean ring between cracked trees and control trees. Differences for wood density between the two groups in relation with position in the ring.

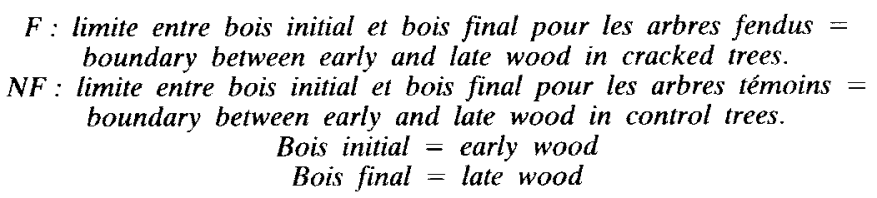


Une explication à l'allure caractéristique de ces 2 profils est peut-être physiologique, avec un rythme de formation du bois d'été différent chez les deux populations; cette hypothèse a été suggérée (Boulet-Gercourt, 1986, op. cit.) mais reste entièrement à confirmer.

\section{Conclusions pratiques et perspectives de recherche}

Ce travail nous aura d'abord permis de montrer, qu'à âge et circonférence égale, la sensibilité à la fente d'Abies grandis semble plus importante pour des arbres ayant des propriétés de leur bois particulières alors que divers paramètres décrivant la forme des arbres ne "répondaient " pas.

Les résultats relatifs à l'angle du fil du bois ont d'abord mis en évidence des valeurs plus élevées chez les arbres fendus. L'infradensité du bois des arbres fendus s'est révélée significativement plus faible et ce, de façon stable sur toute la section de la tige. Ceci a été confirmé par des mesures à la souche. Si elle n'a pas permis une meilleure discrimination des populations d'arbres fendus et non fendus, l'étude microdensitométrique a cependant fait apparaitre que le bois final est plus faible (en pourcentage et surtout en largeur) chez les arbres fendus, a confirmé une relative stabilité des écarts de densité moyenne entre les 2 populations sur l'ensemble des cernes et a localisé de façon précise dans le cerne la zone (limite entre bois initial et bois final) où les différences entre les 2 groupes d'arbres s'accusent tout particulièrement.

En outre, la datation des fentes, très facile sur des sujets abattus, nous a permis de constater que les arbres sont plus sensibles à la fente lors de certaines années qui sembleraient se distinguer des autres par une sécheresse de fin d'été assez importante. Il est remarquable de constater que c'est lors des années à risque que les écarts entre les populations (arbres fendus et non fendus) sont les plus importants sur le plan de la structure du bois.

Tous ces résultats et observations sont cohérents avec ceux des rares publications existant sur le sujet et concernant le Sapin de Vancouver et l'Epicéa commun, deux essences dont tout indique que le comportement est assez voisin eu égard au phénomène.

Ces résultats nous conduisent à proposer des moyens à court terme pour réduire les risques d'apparition du défaut mais surtout suggèrent différentes voies de recherche pluridisciplinaires à plus ou moins long terme permettant en partie de comprendre le phénomène et, par là même, de mieux le juguler.

- Moyens à court terme pour réduire la formation des fentes : il est indiscutable qu'une sélection de population d'arbres ou d'arbres à fil droit et à bois dense devrait statistiquement s'avérer efficace. Cette sélection ne peut d'ailleurs qu'avoir des avantages pour la qualité technologique du produit, ce qui ne gâte rien.

Pour ce qui est de la densité du bois, on sait d'ores et déjà que cette sélection devrait être payante sur Abies grandis puisque les études menées à la Station d'Amélioration des Arbres forestiers de l'I.N.R.A. notamment par Noël (1979) ont mis en évidence une variabilité génétique très significative pour ce critère. Ajoutons que ce 
caractère est en outre très facile à mesurer de manière non destructive. En revanche, la situation est plus difficile pour l'angle du fil du bois car, bien qu'héritable, ce caractère ne peut être mesuré de façon simple à moins de se baser sur une relation grossière pouvant exister avec l'aspect de l'écorce.

Une sélection sur des critères de variation de densité à l'intérieur des cernes, tels que délivrés par la microdensitométrie, ne serait pas réaliste car coûteuse et moins discriminante que sur les critères de densité globale (infradensité).

Ceci n'enlève toutefois rien à l'intérêt de cette technique sur le plan explicatif.

Tout ceci ne doit pas masquer un fait patent à savoir que, dans un peuplement, les sujets fendus font toujours partie des arbres les plus gros ; cela ne surprend d'ailleurs pas puisque l'on sait que, chez Abies grandis comme chez la plupart des Conifères, les arbres les plus gros d'un peuplement équienne ont statistiquement le bois le plus léger. C'est pourquoi en l'absence d'une sélection sur les propriétés du bois, le moyen le plus efficace de limiter le défaut consiste à modérer la croissance individuelle. Ceci pose bien sûr des problèmes.

- Voies de recherche possibles : plusieurs voies sont possibles; elles impliquent des investigations plus ou moins explicatives, plus ou moins liées entre elles et la collaboration de chercheurs de disciplines variées.

Une première voie assez concrète pourrait consister à définir des limites de vitesse de croissance (et le modèle de sylviculture ad hoc) à ne pas dépasser afin que le phénomène «fente » reste marginal. Ces limites pourraient être déterminées dans deux hypothèses de travail suivant que les propriétés internes du bois seraient ou non prises en compte. Ceci permettrait notamment de voir quelle vitesse de croissance individuelle on peut tolérer sans risque lorsque l'on fait un effort de sélection sur la densité du bois par rapport à une situation où seule la vitesse de croissance est prise en compte.

Cela dit, il faut garder à l'esprit le fait qu'une croissance forte et une densité du bois faible ne sont que des critères rendant les arbres sensibles et non la cause du phénomène.

Il resterait bien sûr un champ d'études passionnant à envisager sur le plan physiologique en comparant les deux populations (arbres sensibles, arbres non sensibles) du point de vue de leur comportement hydrique puisque l'hypothèse « fente de sécheresse $»$ se confirme.

C'est pourquoi une autre voie intéressante serait d'examiner l'effet stationnel sur la proportion d'arbres fendus. Dans cette perspective, il serait fort utile de dater les années de formation des fentes pour confirmer ou infirmer les liaisons qui semblent se dégager avec le climat et identifier d'éventuelles stations à risque en prêtant une attention particulière à leur disponibilité en eau. Ceci pourrait être complété par un suivi régulier de l'humidité du bois de certains arbres sensibles en provoquant éventuellement dans leur environnement immédiat une sécheresse artificielle.

Une telle démarche pourrait aussi être adoptée à l'intérieur d'essais de provenances, de clones, ... avec l'avantage cette fois de travailler sur des dispositifs dont l'histoire est parfaitement maîtrisée.

Dans ces deux cas (étude du facteur stationnel ou étude du facteur génétique), il conviendrait de rester attentif au rythme d'activité du cambium, notamment à l'époque de mise en place du bois final. En effet, ce que nous savons, à l'issue de ce travail, sur 
le rôle du bois final quant à la sensibilité de la fente, ne nous interdit pas de penser qu'il peut constituer une barrière à la propagation de fissures. Il n'est pas impossible aussi, ce qui accuserait encore le phénomène, que le comportement physiologique différent des arbres sensibles fasse que le bois d'été se mette en place après la période où l'humidité dans le bois est minimum et où par conséquent les risques de fentes de sécheresse seraient maximum.

Outre ces recherches physiologiques, assez ardues mais dont on sent bien la portée concrète, on peut aussi envisager des études assez fines sur le bois qui nous ont été plus ou moins suggérées au cours de l'interprétation de nos résultats: essais de propagation de microfissures sur bois vert en liaison avec la structure du bois final; mesures de contraintes sur les arbres sur pied en fonction de leur état hydrique au cours de la saison de végétation; essais de résistance en traction tangentielle sur bois vert d'arbres sensibles et non sensibles; recherche de paramètres décrivant la structure du bois plus discriminants que la densité.

Eu égard à la lourdeur de telles expérimentations et à l'imprécision des résultats que l'on peut en attendre, nous doutons qu'il soit possible d'en obtenir des informations aussi fiables et opérationnelles que celles fournies par la densité du bois.

Aussi nous semblerait-il plus efficace d'orienter les recherches futures sur l'importante et intéressante question des fentes des arbres sur pied en direction des premiers thèmes suggérés dans cette conclusion.

Reçu le 27 mars 1987.

Accepté le 28 juillet 1987.

\section{Summary \\ The relationship between wood properties and stem cracks in grand fir Abies grandis (Lindl.)}

Twenty-four pairs of trees, approximately 40 years old, were identified in order to compare wood properties of cracked and sound trees.

Wood characteristics measured were : spiral grain, basic density, wood density variation in ring and between rings.

Many wood properties distinguished the two groups:

- given a similar diameter, the cracked trees have the same height, volume, form and pith eccentricity as the control trees.

Still with a similar diameter :

- the cracked trees have (at $7,5 \mathrm{~m}$ height) more spirality than the control trees (tabl. 1, fig. 3) ;

- most cracks were initiated in 1969 ; the 1969 growth ring of the cracked trees group had the lowest basic wood density (tabl. 2a);

- basic wood density of cracked trees is significantly lower than in control trees. This occurs regularly outwards from the pith (tabl. 2b). Measurements made at ground level confirmed those taken at $6,5 \mathrm{~m}$ (tabl. 2c) :

- the microdensitometric study showed that proportion and chiefly absolute width of late wood were significantly reduced in cracked trees (tabl. 3). Relative regularity of differences in wood density was confirmed from pith to bark (figure 4). Furthermore it allowed us to locate precisely these differences within the growth ring, chiefly at the boundary between early and late wood (fig. 5). 
Some relationships between climate and occurence of the defect were observed through crack dating.

Wood structure differences between cracked and control trees seems to be more important during years in which the defect occured.

These results confirm those of the rare publications in this field.

The authors conclude by proposing means to reduce occurence of the defect and suggesting some basic researches.

Key words : Stem cracks, wood density, microdensitometry, spiral grain, Abies grandis.

\section{Références bibliographiques}

ArChER R.R., 1979. On the distribution of tree growth stresses. Part 3: The case of inclined grain. Wood Science and Technology, 13, 67-68.

Boulet-Gercourt B., 1986. Fentes des arbres chez Abies grandis (Lindl.). Recherche de paramètres de structure du bois pouvant expliquer la sensibilité de certains individus à ce défaut. D.E.A. Sciences $d u$ bois, Station de Recherches sur la Qualité des Bois, C.N.R.F., 119 p.

DAY W.R., 1954. Drought crack of conifers. Imperial Forestry Institute, Oxford. Forest Record $\mathrm{n}^{\circ} 26,39 \mathrm{p}$.

Dietrichson J. et al., 1985. Stem cracks in Norway Spruce (Picea abies (L.) Karst.). Reports of the Norwegian Forest Research Institute, 32 p.

FERRAND J.Ch., 1982. Etude des contraintes de croissance. Deuxième partie : variabilité en forèt des contraintes de croissance du Hêtre (Fagus sylvatica L.). Ann. Sci. For., 39 (3), 187-218.

KeyLWerTh R. 1954. Ein Beitrag zur qualitativen Zuwachanalyse. Die Bestimmung des Raumdichtezahl an klcinen Proben. Holz als Roh- und Werkstoff, 12 (3), 77-83.

LANGlet F., 1985. Les fentes radiales du sapin de Vancouver. Approche du problème en Normandie. Rapport de stage de Technicien supérieur, C.R.P.F. de Normandie, 25 p.

Meuin N., 1984. Influence de la vitesse de croissance sur la qualité du bois de Douglas. Nouvelles observations sur les fissures internes du Douglas. Mémoire de $3^{\mathrm{c}}$ année E.N.I.T.E.F., $140 \mathrm{p}$.

Monchaux P., Nepveu G., 1986. Fentes d'arbres sur pied dans deux plantations d'Epicéa commun (Picea abies (L.) Karst.). Influence de la densité du bois. Ann. Rech. Sylvic., 306-321.

Nö̈. J.C., 1979. Croissance et qualité du bois d'Abies grandis (Lindl.). Etude de la variabilité génétique inter et intrapopulations. Mémoire $3^{\mathrm{c}}$ année E.N.I.T.E.F., $84 \mathrm{p}$.

Polge H., 1982. Quelques observations sur des fissures radiales dans le bois de Douglas. Ann. Sci. For, 39 (4), 399-406.

Polge H., 1984. Nouvelles observations sur les fissures radiales du Douglas. Rev. For. Fr., $X X X V I, 6,453-458$. 